\title{
Atomic Nucleus as Chaotic Quantum Many-Body System
}

\author{
V. ZELEVINSKY \\ Department of Physics and Astronomy and National Superconducting Cyclotron Laboratory, \\ Michigan State University, East Lansing, Michigan 48824-1321, USA
}

\begin{abstract}
Quantum many-body chaos is described as a practical (theoretical, experimental, and computational) instrument in physics of mesoscopic systems of interacting particles. Using mainly nuclear physics applications, it is shown that interactions of constituents create stationary states of high complexity with respect to the nean-field basis with observable properties smoothly changing along the spectrum. Both local Gaussian orthogonal ensemble type features and the global evolution along the spectrum are used to understand the many-body physics and define thermodynamic properties of isolated mesoscopic objects. Among the examples discussed, especially interesting is a chaotic enhancement of weak perturbations illustrated by a large parity violation in neutron resonances on heavy nuclei. Artificially introduced chaotic elements are used to explore the nuclear landscape and predict phase transformations.
\end{abstract}

DOI: 10.12693/APhysPolA.128.1008

PACS: 05.45.Mt, 24.60.Lz, 24.80.+y, 24.10.Pa

\section{Introduction}

Does quantum chaos exist? For a long time this was an interesting pure theoretical question without clear and conclusive answer. Roughly speaking, two schools of thought gave their more or less persuasive answers.

The first viewpoint had more adherents. According to this line of thinking, there exists only classical chaos known from the Poincaré time. In the majority of realistic situations (usually not discussed in the textbooks on classical mechanics), the solutions of well known dynamical equations have extreme sensitivity to initial conditions. Therefore the predictive power of "exact" solutions is limited to the first stage of the evolution of a dynamical system. After that even a tiny round-off error is transformed into the exponentially growing deviation of two phase space trajectories. The long-time evolution of such chaotic systems can be practically described only statistically. In quantum mechanics even the formulation of the dynamical problem in terms of the exact phase space initial conditions is impossible. Moreover, for any finite number of degrees of freedom, quantum dynamics through the Schrödinger equation is formally regular [1]. Therefore it is possible to consider only the traces of classical chaos in quantum systems which are chaotic in the classical limit [2].

Another school of thought [3] is based on the universality and primary character of quantum laws. In this sense the classical chaos can be only the approximate reflection of regularities existing in the quantum world. The justification of this viewpoint can be found in theory of random matrix ensembles [4-6]. In spite of the extremely general character of requirements in formulation of those ensembles, many studies of realistic quantum systems, including those without clear classical analogs, have found striking similarities between the predictions of random matrix theory and actual observable properties. In fact, we are close to reformulating the whole foundation of statistical mechanics in terms of quantum chaos.
One of the best objects for applying the ideas of quantum chaos is given by complex atomic nuclei. This is a typical example from the mesoscopic world. In the mesoscopic universe we have relatively small systems of interacting constituents, where the statistical regularities are already visible but at the same time it is possible to study individual quantum states. It is obvious that complex atoms, molecules and nuclei belong to this world, along with natural and artificial small condensed matter systems. Future quantum computers as systems of qubits interacting on the atomic level also will manifest similar properties. The goal of physics studying this world from the viewpoint of quantum chaos is twofold: to prove that indeed we have quantum chaos in its different manifestations and, which is probably more important, to convert the ideas of quantum chaos in a powerful working instrument.

The first goal was achieved already about twenty years ago, after studies of the so-called Nuclear Data Ensemble [7] and much more detailed analysis of spectroscopy of complex atoms [8] and nuclei $[9,10]$. Below I will give convincing examples from nuclear physics. The second goal is a subject of current work and I will try to show that indeed quantum chaos can be used in nuclear physics problems as a theoretical, experimental, and computational tool.

\section{Nucleus as a testing ground of quantum chaos}

Considering a complex nucleus as a finite Fermi system with strong interactions between the particles living in a mean field we expect that the simple combinatorics of particle-hole excitations lead, already at not very high excitation energy, to large density of many-body quantum states. Then the residual interactions become effectively strong bringing the full mixing of various configurations. If so, the stationary eigenstates are expected to be very complicated superpositions when expressed in the basis of simple mean-field excitations. 
Local spectral statistics is the first appearing and in some sense the weakest signature of quantum chaos. It is predicted in random matrix theory $[6,11]$ that the distribution of the nearest level spacings generated by a time-reversal invariant Hermitian Hamiltonian is given (approximately but with high precision) by the WignerDyson formula for the Gaussian orthogonal ensemble (GOE):

$$
P(s)=\frac{\pi s}{2} \mathrm{e}^{-(\pi / 4) s^{2}},
$$

where $s$ is the level spacing in units of the average local spacing. It is important to guarantee that all levels under consideration belong to the same class in the sense of the exact constants of motion (in nuclei total spin, parity, and isospin). The linear level repulsion at small distances, $P(s) \propto s$, follows from elementary arguments but changes by the quadratic repulsion in the case of violation of time-reversal invariance $(\mathcal{T})$. In practice it is very hard to use this prediction for the search of $\mathcal{T}$-violation because of low statistics of small spacings.

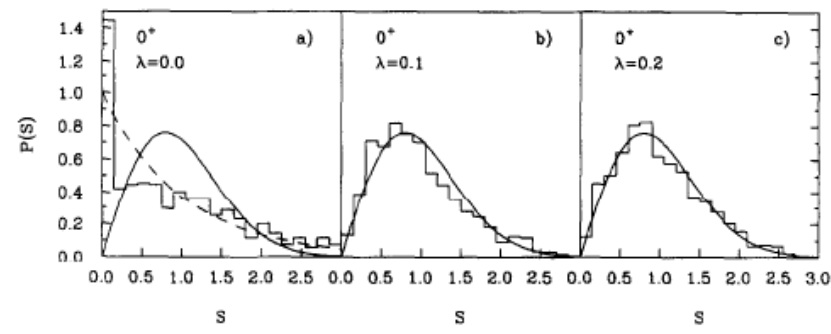

Fig. 1. The level spacing distribution for the levels $J^{\Pi}=0^{+}$in the $s d$-shell model calculation for ${ }^{28} \mathrm{Si}$ [9]. The parameter $\lambda$ artificially scales all offdiagonal matrix elements of the many-body Hamiltonian. The value $\lambda=0$ gives the mean-field approximation with diagonal matrix elements of the residual interaction added in order to remove abundant degeneracies; $\lambda=100 \%$ corresponds to the realistic strength.

The shell-model calculations (exact diagonalization of the full realistic many-body Hamiltonian in the truncated orbital space, in this case the $s d$-model) show, Fig. 1, that the characteristic distribution (1) emerges already at a relatively weak interaction strength $(0.3$ of its realistic value). It is necessary to stress that the used version of the shell model was successfully tested by hundreds of data on energy spectra, transition rates and other observables, so that one can hope that its predictions for high lying nuclear levels are rather reliable, at least in the average sense. Multiple consecutive avoided crossings of levels with the same exact quantum numbers quite naturally establish the "aperiodic crystal" of more or less ordered mutually repelling levels satisfying the distribution (1). Continuing the evolution of the strength $\lambda$ to its realistic value, very soon we see [12] that a more detailed prediction, that of the curvature distribution of the levels $E(\lambda)$, comes to a good agreement with the GOE results.

More information concerning the dynamics one can obtain studying the so-called $\Delta_{3}$ statistics that character- izes the spectral rigidity. This quantity is defined as an average fluctuation of the mean value of the level number in a certain interval of energy,

$$
\Delta_{3}(L)=\frac{1}{L} \min _{a, b}{\overline{\int_{x}^{x+L} \mathrm{~d} \epsilon[\mathcal{N}(\epsilon)-a \epsilon-b]^{2}}}^{x} .
$$

Here the levels are "unfolded" into a constant mean spacing, while the average is taken over the moving energy window of width $L$. In fact, the quantity (2) is determined for a given realization of a random ensemble and as such has its own distribution forming a band, Fig. 2. Using such an ensemble one can simulate the position and the width of this band [13] in the situations of (i) erroneously mixed states which belong to different classes of quantum numbers and/or (ii) of missed levels of a given sequence. Such a statistical analysis for the low-energy neutron resonances in ${ }^{235} \mathrm{U}$ has shown that approximately $4 \%$ of the $s$-wave resonances were missing in the experiments. Another conclusion was that the experiments which do not select a certain value of the compound nucleus spin and therefore can populate both $J=3$ and $J=4$ resonances in ${ }^{236} \mathrm{U}$ give rise to the $\Delta_{3}$ statistics that indeed correspond to the expected statistical ratio $7 / 9$ of the population for these two subsequences.

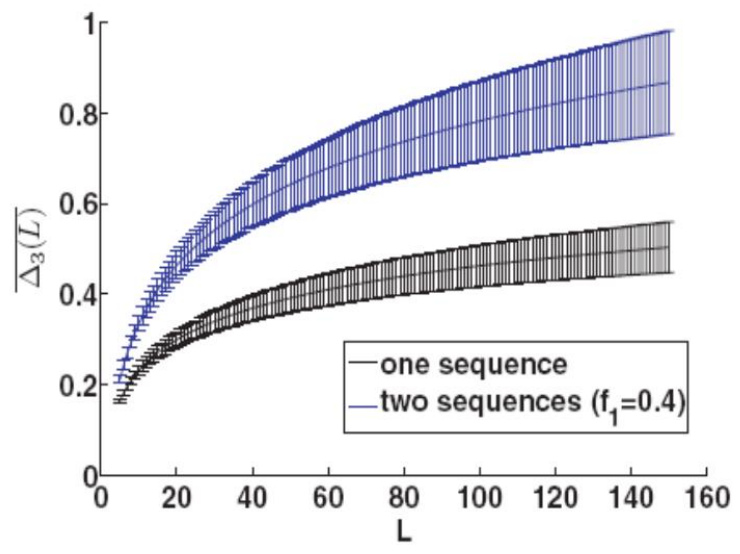

Fig. 2. Bands of spectral rigidity corresponding to a pure sequence and to mixed levels belonging to two families with statistical ratio 0.4 of multiplicities [13].

Of course, the question of the quality of agreement of experimental spectra with the GOE predictions has to be decided by the experiment. Assuming that this agreement indeed takes place, one can use the GOE as an experimental tool for discovery of the fine structure levels invisible in the experiments with poor resolution. An impressive example was given by the analysis [14] of the electron scattering off the ${ }^{208} \mathrm{~Pb}$ nucleus in the region of the giant quadrupole resonance. The missing strength was recovered by a very complicated statistical analysis (two independent procedures which well agree in the end) assuming the existence of hidden levels which approximately obey the GOE statistics. 


\section{Global level density}

It is stressed usually that statistical properties of quantum chaos are local since the application of the GOE to the spectrum as a whole leads to the unphysical semicircle shape of the energy spectrum. Therefore, for example, the spectral rigidity mentioned above makes sense only after the procedure of unfolding. However, the global behavior of the level density is an integral part of any practical calculation of reaction cross-sections, including technological and astrophysical applications. The presence of quantum chaos with its exceedingly complicated stationary wave functions cleans the way to the prediction of the level density on a global scale. The practical algorithm $[15,16]$ is based on the methods of statistical spectroscopy $[17,18]$ that, in turn, can be justified only by the presence of quantum chaos.

The scheme of level density calculations goes as follows. The orbital space is divided into partitions (various distributions of particles over orbitals); in the typical shell-model versions the orbitals are single-particle states in a spherical mean-field potential. Let the dimension of a subspace with conserved given many-body quantum numbers $\alpha$ for a given partition $p$ be $D_{\alpha p}$. Then the level density $\rho_{\alpha}(E)$ with fixed global quantum numbers $\alpha$ is calculated in a function of energy $E$ as

$$
\rho_{\alpha}=\sum_{p} D_{\alpha p} G_{\alpha p}(E)
$$

Here $G_{\alpha p}(E)$ is the Gaussian function whose centroid $E_{\alpha p}$ and the width $\sigma_{\alpha p}$ are determined as the lowest statistical moments of the shell-model Hamiltonian,

$$
E_{\alpha p}=\overline{\langle H\rangle_{\alpha p}}=\frac{1}{D_{\alpha p}} \operatorname{Tr}^{(\alpha p)} H
$$

and

$$
\sigma_{\alpha p}^{2}=\overline{\left\langle H^{2}\right\rangle_{\alpha p}}-E_{\alpha p}^{2} \equiv \frac{1}{D_{\alpha p}} \operatorname{Tr}^{(\alpha p)} H^{2}-E_{\alpha p}^{2},
$$

where the traces are taken over the matrix elements of the Hamiltonian for a given partition and then summed in Eq. (3) over partitions. The calculations are feasible because there is no diagonalization required, the results can be obtained directly from the original matrix, although the derivation of the second moment is rather cumbersome since it includes the matrix elements including different partitions. Higher moments can be calculated in a similar way [17] but it turned out that they are essentially not needed.

The calculations include some technical details necessary for obtaining good results. The Gaussians are modified by introducing the finite range and corresponding renormalization. This cut-off at approximately $3 \sigma$ is motivated by the experience in studying the strength functions [19] from the viewpoint of quantum chaos. If the orbital space includes cross-shell transitions, one needs to eliminate the spurious states related to unphysical admixtures of the center-of-mass motion. A special procedure for this removal was worked out [15]. One has also to mention the problem of the ground state energy that should be known in order to set the whole picture on the correct energy position. There are various practical methods of finding just the ground state energy in each class of states. The chaotic dynamics supplies us with the exponential convergence procedure: it was shown that, for a too large Hamiltonian matrix, after few steps of explicit calculations with a gradually progressing truncation [20], the virtual admixed states are taken from the chaotic region, and the ground state energy (as well as some global observables for this state) can be continued exponentially to the exact result.

To illustrate how the method of moments works, I show three typical calculations. Figure 3 demonstrates the practically perfect agreement of the total level density found in this statistical approach with the result of the exact numerical diagonalization possible in this specific case. This agreement holds in each class of states with given $J^{\Pi} T$ quantum numbers. It also agrees with the low-energy experiments in the cases where the sufficient wealth of reliable data with known quantum numbers of levels is available and we have an appropriate tested version of the shell model. A small deviation in the center of the spectrum can be corrected by the fourth moment of the Hamiltonian but anyway such a high excitation energy is outside of the region where the truncated space of the $s d$-model can aspire to describe the full spectroscopy.
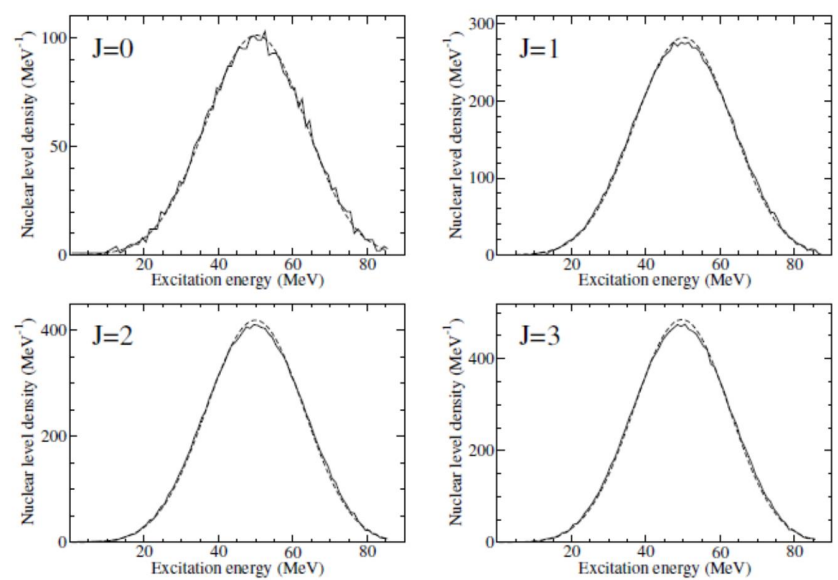

Fig. 3. Comparison of the total level density calculated by the method of moments for ${ }^{28} \mathrm{Si}$ in the $s d$-model with the exact numerical diagonalization.

The standard mean-field combinatorics [21] predicts the level density far from the monotonous increase as a function of energy. The mean-field partitions and pairing effects accounted for in various approaches to this problem strongly modulate the predicted level density, Fig. 4. In contrast to that, the statistical procedure, properly taking into account all shell and pairing effects, produces a smooth curve after including "non-pairing" matrix elements physically corresponding to incoherent collisionlike interactions.

Figure 5 describes the evolution of the level density under influence of such parts of the interaction. Here the 

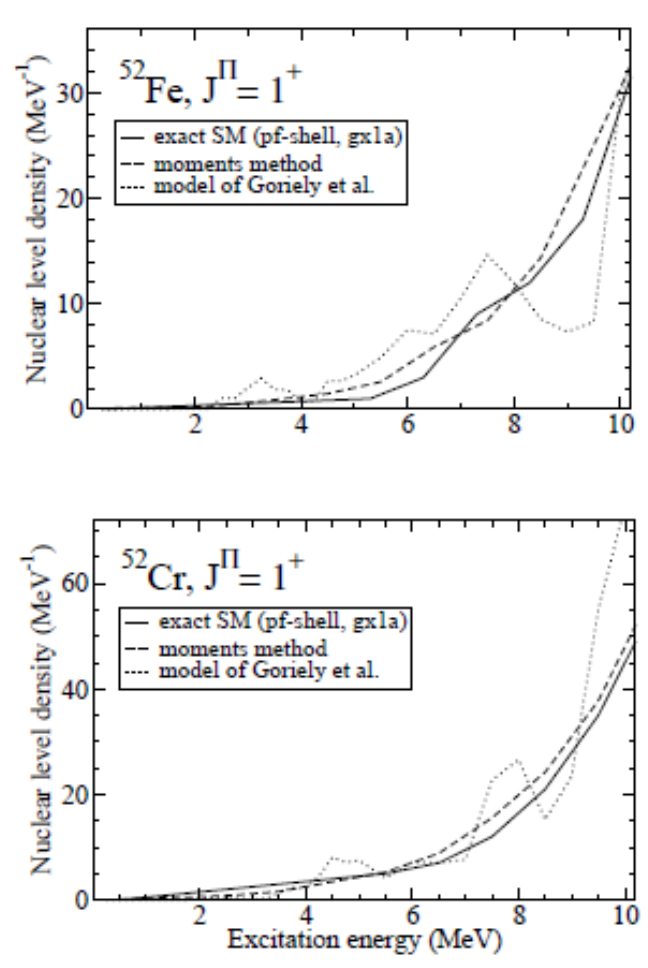

Fig. 4. Comparison of the level density in the method of moments to the results of the mean-field combinatorics $[20]$.

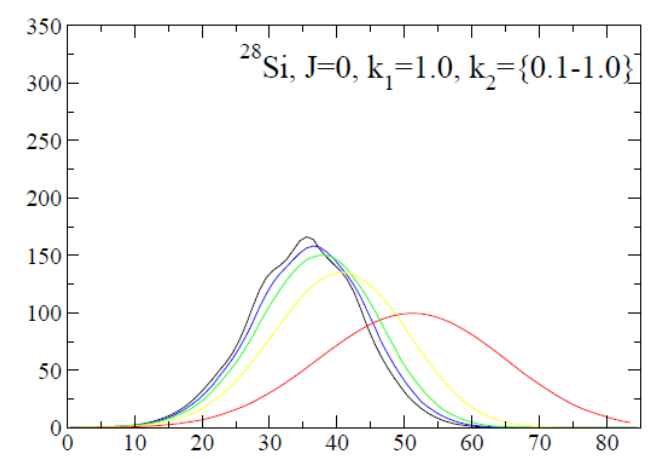

Fig. 5. The influence of various components of the interaction onto the level density. At the pairing strength fixed at its empirical value, consecutive curves reflect the effect of increasing the strength of the non-pairing matrix elements.

shell-model Hamiltonian is presented as

$$
H=H_{\text {meanfield }}+k_{1} V_{\text {pairing }}+k_{2} V_{\text {non-pairing. }} .
$$

The equal importance of different parts of the Hamiltonian in formation of the final level density is clear. In fact, the pairing itself has, at high level density, some chaotic features $[22,23]$. However, they are not sufficient for full chaotic mixing of close in energy wave functions. The irregularities of the level density are almost completely smoothed over in the final level density for the full realistic interaction.

\section{Complexity of wave functions}

The exceedingly complicated nature of the stationary wave functions in the domain of quantum chaos, that actually starts at relatively low excitation energy, around $3-4 \mathrm{MeV}$ in even-even medium nuclei and even earlier in heavy and odd- $A$ nuclei, makes possible to understand and in many cases predict some nontrivial physical phenomena.

It was anticipated long ago [24] that chaotic wave functions in a given energy window "look almost the same". Here an important caveat is that, in contrast to the eigenvalue statistics, the standard measures of complexity are representation-dependent. The frequently used information (Shannon) entropy of individual stationary states $|\alpha\rangle$,

$$
S^{\alpha}=-\sum_{k} w_{k}^{\alpha} \ln w_{k}^{\alpha},
$$

quantifies only the mutual relation between the basis states $|k\rangle$ and the eigenstates of the Hamiltonian

$$
|\alpha\rangle=\sum_{k} C_{k}^{\alpha}|k\rangle, \quad w_{k}^{\alpha}=\left|C_{k}^{\alpha}\right|^{2} .
$$

Still one can argue that if the mean field and corresponding basis $|k\rangle$ are found self-consistently, the main difference of basis states and the majority of genuine stationary states (leaving aside few really collective states) is just due to the chaotic mixing, and therefore the measure (7) indeed reflects the degree of chaotization. The shell model calculations confirm the Gaussian distribution of amplitudes (the Porter-Thomas distribution of probabilities $w^{\alpha}$ ) and absence of correlations between individual components. This was used in the mentioned above analysis [14] of the fine structure invisible in the experiments with insufficient resolution. In the center of the spectrum the entropy (7) is even numerically close to the GOE limit although usually does not quite reach this limit. In fact, many properties follow from the central limit theorem and do not require exact GOE characteristics.

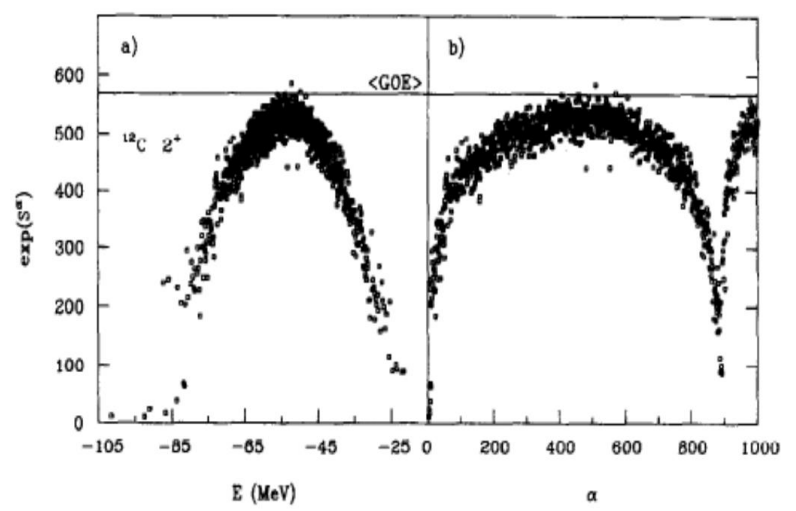

Fig. 6. Information entropy of the states in ${ }^{12} \mathrm{C} \mathrm{nu}-$ cleus in the shell model incorporated cross-shell transitions while the spurious center-of-mass excitations are artificially shifted to high energy. 
The basis-dependent information entropy (8) turns out to be a smooth function of the excitation energy of states $|\alpha\rangle$. It is smart enough, for example, to demonstrate, Fig. 6, the appearance of a new branch of states with exactly the same degree of complexity but shifted to high energy. This happens in the versions of the shell model where the spurious center-of-mass excitations are deliberately moved to high energy and emerge there as another branch of the spectrum with the same degree of complexity as that of the previously found background structure.

\section{Chaotic enhancement of perturbations}

This is probably the most unexpected and practically important aspect of applications of ideas of quantum many-body chaos. It is clear that, due to the very high level density, the mixing of the levels by physical perturbations should be quite different from that in a low-lying energy region. The first impression might be that such a mixing is hardly probable because of absolutely uncorrelated structure of very complicated states adjacent in energy. This is however not the case. We can go through a very crude estimate.

Let an unperturbed state $|\alpha\rangle$, Eq. (8), have a very large number, $N \gg 1$, of principal basis components $|k\rangle$. We assume that the perturbation acts as a simple one- or two-body operator $\hat{Q}$, for example a multipole operator. Such operators, because of selection rules, have a finite (rather small) number $n$ of non-zero matrix elements between the basis states with a characteristic value $q$ of these matrix elements. Since the typical amplitudes $C_{k}^{\alpha}$ are of the order of $1 / \sqrt{N}$, the matrix element between the complex and basis states can be estimated as

$$
\langle k|\hat{Q}| \alpha\rangle=\sum_{l} C_{l}^{\alpha} Q_{k l} \simeq \frac{q n}{\sqrt{N}} .
$$

In the same way, the matrix element between two chaotic states close in energy (and therefore of the same degree of complexity $N$ ) is evaluated by order of magnitude as

$$
\langle\beta|\hat{Q}| \alpha\rangle=\sum_{\alpha \beta} C_{k}^{\beta *} C_{l}^{\alpha} Q_{k l} \simeq \frac{q n}{\sqrt{N}},
$$

where we took into account that one summation is taken care of by the estimate (9) and another one, due to the chaoticity of signs of the amplitudes $C$, gives the factor $\sqrt{N}$ instead of $N$.

Thus, the matrix elements of simple operators between close chaotic states are suppressed only by a factor $1 / \sqrt{N}$. But we are interested in the response of the system in the region of very high level density. The typical level spacings are here smaller by a factor of $\sim N$ compared to the region of low-lying "simple" states. As a result, the mixing of complicated states is predicted to be enhanced by a factor $\sim \sqrt{N}$. Such estimates apparently were first suggested by Blin-Stoyle [25] and, in more detailed form, by Sushkov and Flambaum [26], specifically for the enhancement of parity violation in nuclei.

Parity violation in nuclei occurs due to the weak forces between the nucleons. The effective interaction in the mean field description is given by the "simple" local operator proportional to $G \rho(\boldsymbol{r})(\boldsymbol{\sigma} \cdot \boldsymbol{p})$, where $G$ is the Fermi constant, $\rho(\boldsymbol{r})$ - local nuclear density and $\boldsymbol{p}$ - the nucleon momentum operator. On the level of simple meanfield states this operator mixes single-particle orbitals of opposite parity providing the parity violating admixture of the order $10^{-7}$ or $10^{-8}$. A very large parity violation, up to $10 \%$ in some cases, was observed in the resonance scattering of longitudinally polarized neutrons in Dubna [27] and Los Alamos [28]. The ratio

$$
\mathcal{P}=\frac{\sigma^{+}-\sigma^{-}}{\sigma^{+}+\sigma^{-}}
$$

for corresponding cross-sections should vanish if the parity is strictly conserved. In Fig. 7 we see a very large effect at a resonance $E_{n}=63.5 \mathrm{eV}$ in ${ }^{238} \mathrm{U}$. At the neutron resonance energy, the complexity parameter $N \approx 10^{6}$ so that we expect the enhancement $\sqrt{N} \approx 10^{3}$. In addition, the so-called kinematic factor leads to the further enhancement, the ratio of the partial neutron widths for the mixture of the $p$ - and $s$-resonances, $\Gamma_{s} / \Gamma_{p} \approx 10^{3}$. Altogether we have the enhancement by 6 orders of magnitude that allows one to see the parity violation by naked eye, Fig. 7.

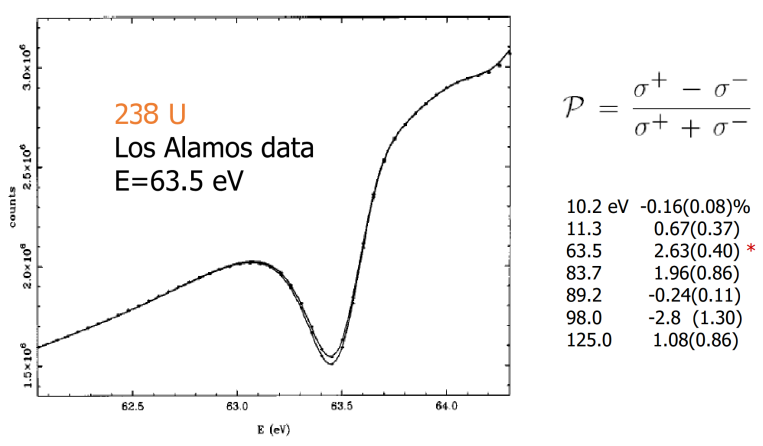

Fig. 7. The ratio $\mathcal{P}$ for cross-sections $\sigma^{ \pm}$in the resonance scattering of neutrons with opposite helicities [28]. The right column gives $\mathcal{P}$ for different resonances in ${ }^{238} \mathrm{U}$.

Another observable, the asymmetry of the motion of the fission fragments with respect to the spin of the initial neutron, was even more unexpected [29]. This asymmetry in the experiment is enhanced "only" by 3-4 orders of magnitude being of the same magnitude as some asymmetries allowed by parity conservation (here there is no kinematic enhancement). The adiabatic process of neutron fission goes through a pear-shaped configuration that corresponds, with parity conserved, to parity doublets. A small parity violation makes the doublet components not equivalent creating a fragment asymmetry which is enhanced by the chaotic mechanism. This can happen only at the "hot stage" of the reaction where the strong mixing is active. The further evolution to the scission point preserves the asymmetry. Then one can make a crucial prediction that the parity violation cannot depend on the details of mass distribution or division of 
kinetic energy between the fragments - these characteristics are decided later. The Grenoble experiments [30] clearly show that the asymmetry on the level of $10^{-3}$ or $10^{-4}$ indeed is practically constant for all numerous fission channels: the enhancement is predetermined by the chaotic stage being independent of what happens later in the fission process, Fig. 8.
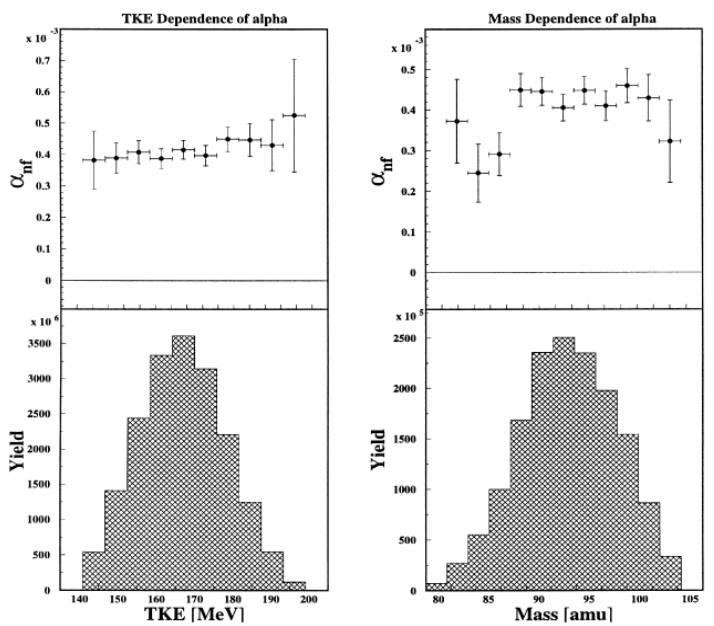

Fig. 8. The asymmetry of fragments in fission of ${ }^{233} \mathrm{U}$ by cold polarized neutrons, $\propto\left(\boldsymbol{\sigma}_{n} \cdot \boldsymbol{p}_{\text {fragment }}\right)$, according to Ref. [30] is constant for different channels (mass and total kinetic energy distributions).

Qualitatively similar enhancement effects work in many other situations, for example, in mixing of superdeformed bands with bands of "normal" deformation [31-33]. This physics also defines the excitation energy of structure isomers that would allow their mixing with other states and trigger their decay [34].

\section{Chaos and thermalization}

From the first years of nuclear physics, highly excited nuclear states have been discussed not in terms of individual wave functions but rather using the ideas and terminology of statistical physics and thermodynamics (temperature, entropy, evaporation etc.). Are those two languages mutually exclusive? Complementary? Equivalent?

The rich experience of the last two decades allows us to start a reasonable discussion of these questions. One obstacle on this path is a standard prejudice that the thermodynamic description is valid only on a macroscopic scale that corresponds to the so-called thermodynamic limit of large volume and large particle number with their ratio (density) fixed. On the other hand, we can look carefully at the statistical results for the set of individual stationary wave functions in the exact shell-model calculations. Of course, the properties of the system which are statistical by their construction, as the global level density, are smooth functions of energy and maybe of other constants of motion. But we immediately see also that such characteristics of the eigenstates as, for example, information entropy, found not globally but for each individual state, are very smooth functions of excitation energy. Therefore they can be considered as thermodynamic variables uniquely defined up to fluctuations which, indeed, depend on the dimension of space.

This is an appropriate moment in order to apply to classics of theoretical physics. In the book of Statistical Physics by Landau and Lifshitz [35] we read: "It may again be mentioned that, according to the fundamental principles of statistical physics, the result of the averaging is independent of whether it is done mechanically over the exact wave function of the stationary state of the system or statistically by means of the Gibbs distribution. The only difference is that in the former case the result is expressed in terms of the energy of the body, and in the latter case as a function of its temperature". This point of view was developed in the detailed practical analysis of spectra for complex atoms [8], nuclei [9], and matrix models [36]. The mechanism that allows us to obtain the equivalent information from a typical (but very complicated) wave function is quantum chaos that makes all individual states in some energy interval "look the same" [24, 37-40]. In the recent literature, this idea sometimes is called the eigenstate thermalization hypothesis.

Here I give just an example of how thermodynamic ideas and corresponding instruments agree with the results of the exact solution of the quantum many-body problem for a mesoscopic system, such as an atomic nucleus. The level density found by the full diagonalization in a truncated space or with the help of the method of moments reveals a very fast growth to the centroid and then almost symmetric fall. Here one can define a global thermodynamic temperature as

$$
T_{\mathrm{th}}=\left(\frac{\mathrm{d} \ln \rho(E)}{\mathrm{d} E}\right)^{-1},
$$

essentially through thermodynamic entropy $S_{\mathrm{th}}=-\ln \rho$. For the curve $\rho(E)$ close to the Gaussian with a width $\sigma_{E}$, this temperature goes from positive to negative infinity at the centroid $E=E_{c}$,

$$
T_{\mathrm{th}}=\frac{\sigma_{E}^{2}}{E-E_{c}} .
$$

Other definitions of the effective temperature are also possible. Assuming the Landau ideology of Fermi-liquid, we can consider the system as a conglomerate of fermionic quasiparticles distributed over orbitals with energies $\epsilon_{i}$ according to

$$
n_{i}^{\alpha}=\frac{1}{1+\exp \left(\left(\epsilon_{i}-\mu^{\alpha}\right) / T_{\text {s.p. }}^{\alpha}\right)},
$$

where we calculate the occupancies $n_{i}^{\alpha}$ for each individ$u a l$ eigenstate $|\alpha\rangle$ and try to fit the chemical potential $\mu$ and the effective single-particle temperature $T_{\text {s.p. }}$ for each state separately in order to extract the evolution of these thermodynamic parameters along the spectrum of the system. Finally it is also interesting to find the 
effective information entropy (7) and corresponding informational temperature similarly to Eq. (12),

$$
T_{\mathrm{inf}}^{\alpha}=\left(\frac{\mathrm{d} S^{\alpha}}{\mathrm{d} E}\right)^{-1},
$$

again for each individual stationary state $|\alpha\rangle$.

The analysis shows that all definitions of temperature as a function of excitation energy practically coincide if we use the appropriate scales ("thermometers"). For example, the two lines for positive and negative thermodynamic temperature branches, Eq. (13), are indeed the loci of the points corresponding to quasiparticle temperatures extracted according to Eq. (14) for all individual states (the chemical potential is almost constant), Fig. 9.

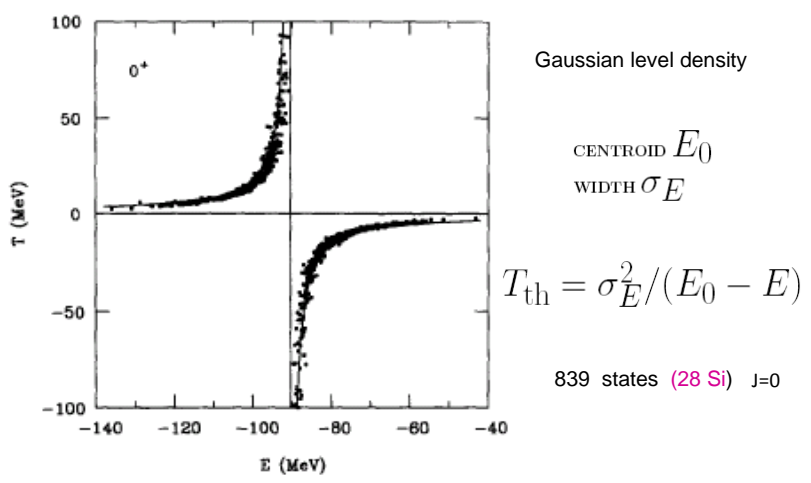

Fig. 9. Effective temperature $T_{\text {s.p. }}$ of individual states $J=0$ in the shell model calculation, see Eq. (13), for ${ }^{28} \mathrm{Si}$, dots, and the global thermodynamic temperature $T_{\mathrm{th}}$, lines, found from the level density.

It is necessary to stress that the agreement of comparisons analogous to that in Fig. 9 can be easily destroyed by the choice of a wrong thermometer. It was said already that the information entropy and therefore temperature characterizes our system with respect to a selected basis of our representation. The thermodynamic definition (12) is basis-independent. The convincing agreement of different definitions, as in Fig. 9, corresponds to the empirical shell-model mean field and the residual interaction consistent with this field. If the residual interaction is made "by hand" much stronger, the quasiparticle evolution in the spirit of the Fermi liquid disappears, and the system essentially is all the time heated to infinite temperature. However the possibility to vary the interaction and to study the change of dynamical features can also be instructive as we briefly explain in the next section.

\section{Artificial chaos}

New theoretical tools appear along with the possibility to randomize the Hamiltonian in order to better understand the effects of its different parts in their common action. A very promising tool can be formulated in terms of correlational entropy [41].

For any eigenstate $|\alpha\rangle$ we define its density matrix $\rho^{\alpha}$,

$\left(\rho^{\alpha}\right)_{k l}=C_{l}^{\alpha *} C_{k}^{\alpha}$,

so that the expectation value of any operator $\hat{Q}$ in the state $|\alpha\rangle$ be

$$
\langle\alpha|\hat{Q}| \alpha\rangle=\sum_{k l} C_{l}^{\alpha *} C_{k}^{\alpha} Q_{l k}=\operatorname{Tr}\left(Q \rho^{\alpha}\right) .
$$

Here the result is invariant with respect to the choice of the original basis but does not provide any new information. Now we redefine the density matrix as an average over some random ensemble,

$$
\left(\rho^{\alpha}\right)_{k l} \Rightarrow \overline{C_{l}^{\alpha *} C_{k}^{\alpha}}
$$

but still for a given state $|\alpha\rangle$ that can be followed continuously varying the ensemble parameters. While the trivial matrix (16) had eigenvalues equal to 1 for the state $|\alpha\rangle$ and 0 for any orthogonal state, the new matrix (18) has eigenvalues between 0 and 1 and can be diagonalized in its own eigenbasis (canonical, or pointer basis).

For the new density matrix we can define the new entropy,

$$
S_{\text {corr }}^{\alpha}=-\operatorname{Tr} \ln \rho^{\alpha}, '
$$

again for any specific state. This member of the broad entropy family is invariant under basis transformation. In distinction to the previous entropies discussed in our thermodynamic part this quantity shows the response of the system to the parameter variations. There is a great freedom in formulating wise questions and try to answer them with the specific choices of the ensemble for this purpose. The simplest and physically clearest way is to vary randomly the parameters of a realistic Hamiltonian and look for the signatures of possible phase transitions in the structure of the system. If the artificial chaotic ensemble contains variables randomly covering the region of structural changes, the fluctuations grow, and the correlational entropy (19) obediently displays a maximum that gives the signal of restructuring.

In this way we have studied the interplay of the standard isovector pairing competing with the quasideuteron isoscalar pairing in sd-nuclei [42]. The correlational entropy in the ground state of the nucleus reveals a typical maximum at the values of the isoscalar pairing constant that are approximately three times greater than what we have in a realistic Hamiltonian. The conclusion is that the quasideuteron correlations which are certainly present in light odd-odd nuclei give way to the normal pairing in heavier, especially even-even, nuclei.

Making matrix elements of the residual interaction (all or some of them) random quantities, we can find the ranges of the parameters responsible for specific observable properties. In this way, the question of the predominance of prolate deformation in nuclear ground states was studied [43] (among stable nuclei there is just one case, ${ }^{12} \mathrm{C}$, that shows up oblate deformation, mainly due to the admixtures of $3 \alpha$ cluster structure). Among the guilty matrix elements (in the $p f$-shell) are those which mix the $p$ and $f$-orbitals in the nuclei before the midshell creating prolate deformation through populated levels with small angular momentum projection onto the symmetry axis. In the end of the shell the orbitals with large projections and therefore preferred oblate deformation would be energetically favorable but they are defeated by the levels coming from upper shells. 
Such methods of introducing the artificial chaos and trying to understand emerging physics still leave unanswered several profound questions. One of them is related to an accidental discovery [44] that the rotationally invariant residual interactions with random by magnitude matrix elements produce predominantly the ground state of zero total spin. This happens in spite of a rather low fraction of zero-spin states in the total Hilbert space: the ensemble averaging prefer the lowest symmetry of angular momentum coupling. Here we deal with the new element of the whole theory of quantum chaos correlations of subensembles of states with different values of constants of motion but governed by the same many-body Hamiltonian. The idea that the mean field arises from the chaotic interactions accumulating the regular features of the dynamics [45-47] provides some understanding to this set of observations but still leaves many questions not answered, especially about considerable probability of typical collective phenomena (vibrations and rotations) appearing from random interactions with rather high probability.

\section{Conclusion}

The short journey through the chaotic jungles of nuclear many-body physics has shown that the ideas of quantum chaos bring new instruments to serve the progress of our understanding. These ideas were first born in relation to the nuclear problems and then penetrated the quantum physics in general, especially the mesoscopic domain.

We tried to demonstrate with typical examples that physics of quantum chaos became really much more rich. We can recall experimental tools (extraction of invisible fine structure in experiments with insufficient resolution), computational methods (method of moments for finding the global level density, exponential convergence of ground state energy and other observables, exploration of the many-body landscape with random interactions, etc.), new theoretical approaches (thermalization of small isolated systems, chaotic enhancement of weak perturbations, artificial chaos etc.). Many questions are still not fully understood and future work in these directions is absolutely necessary.

Almost completely left outside of this story is the chapter on quantum chaos in reactions and continuum states which were mentioned only in the section on level density and in explanation of the processes with strong parity violation. This part requires a detailed explanation on its own, starting from the Bohr concept of compound nuclei. The statistical search for the invisible fine structure states requires in fact two conditions: the spacing $D$ should be not only small compared to the energy resolution of the experiment but, on the other hand, it should be large compared to the typical decay widths $\Gamma$ of the continuum states contributing to the giant resonance. Very new physics related to overlapping resonances emerges in the opposite limit of $\Gamma \geq D$ and different terminology becomes necessary (statistics of lifetimes, superradiance, transmission, cross-sections and so on). Many results turn out to be common for nuclear physics and condensed matter physics of disordered materials [48]. Here I limit myself with few references [49-56].

\section{Acknowledgments}

This article is based on the results of the many-year work by a large group of great scientists; I can only express my deep gratitude listing them in alphabetical order: Naftali Auerbach, Gennady Berman, B. Alex Brown, Luca Celardo, Victor Flambaum, Njema Frazier, Yakov Greenberg, Mihai Horoi, Felix Izrailev, Lev Kaplan, Declan Mulhall, Roman Sen'kov, Valentin Sokolov, Suren Sorathia, Amin Tayebi, Alexander Volya. Amin Tayebi was also very helpful in preparing this article. I am thankful to L. Sirko and S. Bauch for hospitality in Warsaw.

The support from the NSF grant PHY-1404442 is gratefully acknowledged.

\section{References}

[1] V. Zelevinsky, Quantum Physics, Vol. II, Wiley-VCH, Weinheim 2011.

[2] F. Haake, Quantum Signatures of Chaos, Springer, New York 1991.

[3] B.V. Chirikov, F.M.Izrailev, D.L. Shepelyansky, Physica D Nonlin. Phenom. 33, 77 (1988).

[4] E.P. Wigner, Ann. Math. 62, 548 (1955).

[5] Selected Papers of Freeman Dyson with Commentary, American Mathematical Society, Providence 1996; F.J. Dyson, M.L. Mehta, J. Math. Phys. 4, 701 (1963).

[6] M.L. Mehta, Random Matrices, 3rd ed., Elsevier, Amsterdam 2004

[7] O. Bohigas, M.J. Giannoni, C. Schmit, Phys. Rev. Lett. 52, 1 (1984).

[8] V.V. Flambaum, A.A. Gribakina, G.F. Gribakin, M.G. Kozlov, Phys. Rev. A 50, 267 (1994).

[9] V. Zelevinsky, B.A. Brown, N. Frazier, M. Horoi, Phys. Rep. 276, 315 (1996).

[10] V. Zelevinsky, Annu. Rev. Nucl. Part. Sci. 46, 237 (1996).

[11] A. Bohr, B. Mottelson, Nuclear Structure, Vol. 1, Benjamin, New York 1969.

[12] D. Kusnezov, B.A. Brown, V. Zelevinsky, Phys. Lett. B 385, 5 (1996).

[13] D. Mulhall, Z. Huard, V. Zelevinsky, Phys. Rev. C 76, 064611 (2007).

[14] G. Kilgus, G. Kührer, S. Müller, A. Richter, W. Knüpfer, Z. Phys. A 326, 41 (1987.

[15] R.A. Sen'kov, M. Horoi, V. Zelevinsky, Phys. Lett. B 702, 413 (2011).

[16] R.A. Sen'kov, M. Horoi, V. Zelevinsky, Comp. Phys. Commun. 184, 215 (2013).

[17] S.S.M. Wong, Nuclear Statistical Spectroscopy, University Press, Oxford 1986. 
[18] Spectral Distributions in Nuclei and Statistical Spectroscopy, Eds. V.K.B. Kota, R.U. Haq, World Sci., Singapore 2010.

[19] N. Frazier, B.A. Brown, V. Zelevinsky, Phys. Rev. C 54, 1665 (1996), reprinted in Ref. [18], p. 557.

[20] M. Horoi, A. Volya, V. Zelevinsky, Phys. Rev. Lett. 82, 2064 (1999); M. Horoi, B.A. Brown, V. Zelevinsky, Phys. Rev. C 65, 027303 (2002); Phys. Rev. C 67, 034303 (2003).

[21] S. Hilaire, M. Girod, S. Goriely, A.J. Koning, Phys. Rev. C 86, 064317 (2012).

[22] A. Volya, V. Zelevinsky, B.A. Brown, Phys. Rev. C 65, 054312 (2002)

[23] J.R. Armstrong, S. Åberg, S.M. Reimann, V.G. Zelevinsky, Phys. Rev. E 86, 066204 (2012).

[24] I.C. Percival, J. Phys. B 6, L229 (1973).

[25] R.J. Blin-Stoyle, Phys. Rev. 120, 181 (1960).

[26] O.P. Sushkov, V.V. Flambaum, Sov. Phys. Usp. 25, 1 (1982)

[27] V.P. Alfimenkov, S.V. Borzakov, V. Van Thuan, Yu.D. Mareev, L.B. Pikelner, A.Z. Khrykin, E.I. Sharapov, Nucl. Phys. A 398, 93 (1983).

[28] J.D. Bowman, G.T. Garvey, M.B. Johnson, G.E. Mitchell, Annu. Rev. Nucl. Part. Sci. 43, 829 (1993).

[29] G.V. Danilyan, Sov. Phys. Usp. 23, 323 (1980).

[30] A. Koetzle, P. Jesinger, F. Goennenwein, G.A. Petrov, V.I. Petrova, A.M. Gagarsky, G. Danilyan, O. Zimmer, V.V. Nesvizhevsky, Nucl. Instrum. Methods Phys. Res. A 440, 750 (2000).

[31] R. Krücken, A. Dewald, P. Sala, C. Meier, H. Tiesler, J. Altmann, K.O. Zell, P. von Brentano, D. Bazzacco, C. Rossi-Alvarez, R. Burch, R. Menegazzo, G. de Angelis, G. Maron, M. de Poli, Phys. Rev. Lett. 73 , 3359 (1994).

[32] J.-Z. Gu, H.A. Weidenmueller, Nucl. Phys. A 660, 197 (1999).

[33] S. Åberg, Phys. Rev. Lett. 82, 299 (1999).

[34] N. Auerbach, V. Zelevinsky, Phys. Rev. C 90, 034315 (2014).

[35] L.D. Landau, E.M. Lifshitz, Statistical Physics, Pergamon Press, Oxford 1958.

[36] V.V. Flambaum, F.M. Izrailev, Phys. Rev. E 56 5144 (1997)
[37] A. Peres, Phys. Rev. A 30, 504 (1984).

[38] J.M. Deutsch, Phys. Rev. A 43, 2046 (1991).

[39] M. Srednicki, Phys. Rev. E 50, 888 (1994).

[40] M. Rigol, V. Dunjko, M. Olshanii, Nature 452, 854 (2008).

[41] V.V. Sokolov, B.A. Brown, V. Zelevinsky, Phys. Rev. E 58, 56 (1998).

[42] A. Volya, V. Zelevinsky, Phys. Lett. B 574, 27 (2003).

[43] M. Horoi, V. Zelevinsky, Phys. Rev. C 81, 034306 (2010).

[44] C.W. Johnson, G.F. Bertsch, D.J. Dean, Phys. Rev. Lett. 80, 2749 (1998).

[45] V.G. Zelevinsky, Nucl. Phys. A 555, 109 (1993).

[46] D. Mulhall, A. Volya, V. Zelevinsky, Phys. Rev. Lett. 85, 4016 (2000).

[47] V. Zelevinsky, A. Volya, Phys. Rep. 391, 311 (2004).

[48] C.W.J. Beenakker, Rev. Mod. Phys. 69, 731 (1997).

[49] V.V. Sokolov, V.G. Zelevinsky, Phys. Lett. B 202 10 (1988); Nucl. Phys. A 504, 562 (1989).

[50] V.V. Sokolov, V.G. Zelevinsky, Ann. Phys. 216, 323 (1992).

[51] V.V. Sokolov, V. Zelevinsky, Phys. Rev. C 56, 311 (1997).

[52] A. Volya, V. Zelevinsky, in: Nuclei and Mesoscopic Physics: WNMP 2004, Ed. V. Zelevinsky, AIP, Melville 2005, p. 229.

[53] N. Auerbach, V. Zelevinsky, Rep. Prog. Phys. $\mathbf{7 4}$ 106301 (2011).

[54] G.L. Celardo, F.M. Izrailev, V.G. Zelevinsky, G.P. Berman, Phys. Rev. E 76, 031119 (2007); Phys. Lett. B 659, 170 (2008).

[55] S. Sorathia, F.M. Izrailev, G.L. Celardo, V.G. Zelevinsky, G.P. Berman, EPL 88, 27003 (2009); G.L. Celardo, A.M. Smith, S. Sorathia, V.G. Zelevinsky, R.A. Sen'kov, L. Kaplan, Phys. Rev. B 82, 165437 (2010); S. Sorathia, F.M. Izrailev, V.G. Zelevinsky, G.L. Celardo, Phys. Rev. E 86, 011142 (2012).

[56] Ya.S. Greenberg, C. Merrigan, A. Tayebi, V. Zelevinsky, Eur. Phys. J. B 86, 368 (2013). 